\title{
Layers Method Implementation for Rubik's Cube Solution
}

\author{
${ }^{1}$ Robbi Rahim \\ ${ }^{1}$ Department of Informatics, Institut Teknologi Medan, \\ Indonesia \\ usurobbi85@zoho.com \\ ${ }^{2}$ Ronal Hadi, ${ }^{3}$ Rasyidah \\ ${ }^{2,3}$ Department of Information Technology, Politeknik Negeri \\ Padang, Indonesia \\ ronalhadi@polinpdg.ac.id, rasyidah@polinpdg.ac.id \\ ${ }^{4}$ Heri Nurdiyanto \\ ${ }^{4}$ Department of Informatics, STMIK Dharma Wacana, \\ Metro Lampung, Indonesia \\ herinurdiyanto@gmail.com \\ ${ }^{5}$ Darmawan Napitupulu \\ ${ }^{5}$ Research Center for Quality System and Testing \\ Technology, Indonesian Institute of Sciences, Indonesia \\ darwan.na70@gmail.com
}

\author{
${ }^{6}$ Ansari Saleh Ahmar \\ ${ }^{6}$ Department of Statistics, Universitas Negeri Makasar, \\ Indonesia \\ ansarisaleh@unm.ac.id \\ ${ }^{7}$ Leon Andretti Abdillah \\ ${ }^{6}$ Department of Information System, Universitas Bina \\ Darma, Indonesia \\ leon.abdillah@yahoo.com \\ ${ }^{8}$ Dahlan Abdullah \\ ${ }^{8}$ Department of Informatics, Universitas Malikussaleh, \\ Indonesia \\ dahlan@unimal.ac.id \\ ${ }^{9}$ Muhammad Ikhsan Setiawan \\ ${ }^{9}$ Department of Civil Engineering, Narotama University, \\ Indonesia \\ ikhsan.setiawan@narotama.ac.id \\ ${ }^{10}$ Janner Simarmata \\ ${ }^{10}$ Universitas Negeri Medan, Indonesia \\ jannersimarmata@unimed.ac.id
}

\begin{abstract}
Rubik's cube is a reasonably favorite cube game until today with many variations of the cube being developed to increase the difficulty of players; the $3 \times 3$-sized Rubik's cube is most popular use for beginner and also for an expert. In this article will be presented step by step how to solve Rubik's cube $3 \times 3$ by using layers method and show each detail side of Rubik's cube, to facilitate new users to use and learn Rubik's cube an application made by applying layers method, and this app could be used as medium for learning for Rubik's cube.
\end{abstract}

Keywords-Rubik's Cube, Layers Method, Rubik's Application

\section{INTRODUCTION}

Rubik's cube[1]-[3] is a game of agility and algorithmic calculation to complete the game. Rubik's cube seen as a decisive game, affordable, motor training and memory and train logic geometry for Rubik's player game. Rubik's is a cube-shaped mechanical puzzle game that has colors on each side[1], divided into several small squares that have the same prefix color group for each side such as red, yellow, green, blue, and white[3], [4], which can be rotated in a way that it produces random balances on each side.
Rubik's cube can be solved by using a specific algorithm that must be known by Rubik's player to arrange Rubik's piece according to the color group[4], [5], one of the algorithms that can use is layer by layer (layers) algorithm[6]. Layers Algorithm is a technique used by novice players who want to finish Rubik's cube game. Layer Algorithms can solve a random Rubik's Cube by using step in a "layer by layer" on each side of Rubik's cube[6], [7]. Layers can solve Rubik's Cube starting first, second, and the third layer.

Implementation of layers algorithm in the application is supposed to allow accomplishment 3x3 Rubik's cube game with randomly generated conditions with varying difficulty levels, the solution produced can be followed by the player to complete the Rubik's cube game.

\section{Methodology}

\section{A. Rubik's Cube}

Rubik's Cube is a three-dimensional mechanical puzzle, discovered in 1974 by a Hungarian sculptor and professor of architecture named Erno Rubik[2], [8]-[10]. In the mid-1970s, 
Erno Rubik wanted to model as a learning tool to help his students understand three-dimensional geometry and eventually become the world's best-selling puzzle toy[5], [11], [12], Fig. 1.

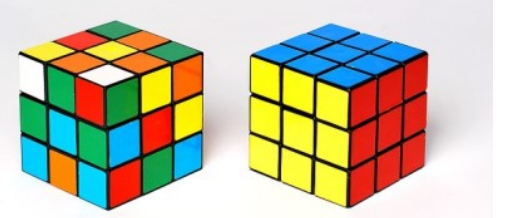

Fig. 1. Simple Cryptography Process

The usual terminology used to describe a Rubik's Cube is as follows:

1. Rubik's Cube consists of six sides: front, back, left, right, up (up) and down (down) side on Rubik's

2. The rotating motion of the Rubik's side of the Cube is clockwise symbolized by the first letter of the side name in English. For counter-clockwise, quotes (") are added. For example, $\mathrm{F}$ means rotating the front side clockwise, while L "means rotating the left side in opposite direction with the clock, Fig 2.
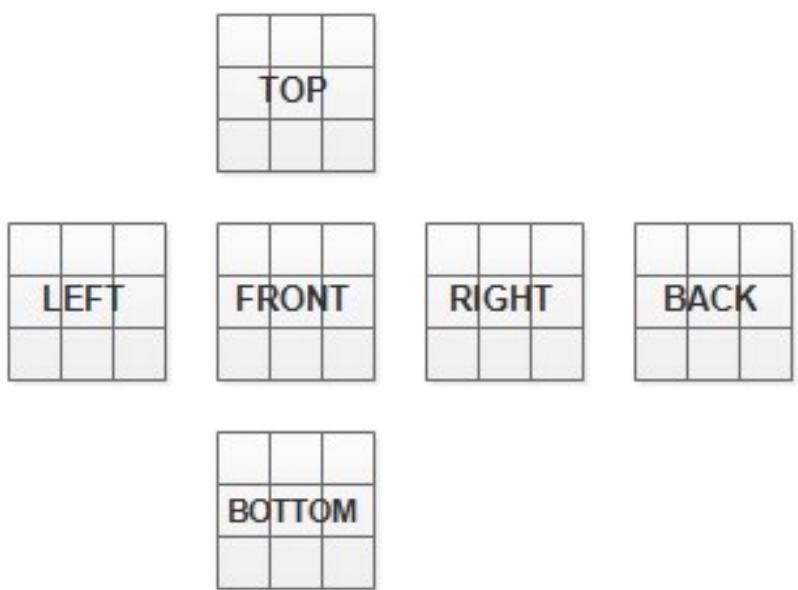

Fig. 2. Two Dimension Rubik's Cube

\section{B. Layer by Layer Algorithm}

The layer by layer algorithm is a variant of the greedy algorithm[6], [7] to solve the Rubik game and its implementation to complete the Rubik's cube using application. Some steps in the layer by layer provide an alternative solution for every "possible" condition occurring on a cube. Each state has a key that must be known by the player, the requirement for that key will be created by using a layer by layer algorithm, and the principal method of solving Rubik's cube by applying a layer by layer algorithm will always go through the following six stages[3]:
1. Form cross on the first layer. The key is to make sure the edge of each cross has the same color as the center of each side.

2. Finishing the first layer, there is no particular step here but it is precisely this that makes the completion stage can be extended if you do not do the calculation from the beginning.

3. Solving the second layer, Steps to note only steps to finish the edge of the second layer. On average each stage of each edge takes nine steps.

4. Form cross on the last layer. There are two steps to consider in the formation of the final cross-plane, i.e., if some of the crosses is formed on two sides with the condition (1) two adjacent sides are formed, or (2) two opposite sides are formed. At this stage, there is no need to direct the cross with the appropriate color, merely crossregardless its color.

5. Adjust the color match of the cross that has been formed. One step is needed to solve it.

6. Set each corner on the last layer. This setting requires at least two steps to adjust the position at the correct angle and adjust the color at each angle.

\section{Proposed METHOD}

Under completion conditions using layer-by-layer algorithms, any movement performed on the Rubik's layer will be stored in an Array. This array will be used to check whether it has solved the random Rubik's cube or not. Players can complete a cube that is scrambled then the system will check the Rubik cube has been arranged each color on every side. The un-random Rubik's has the six sides of the color still organized as in Fig. 3.
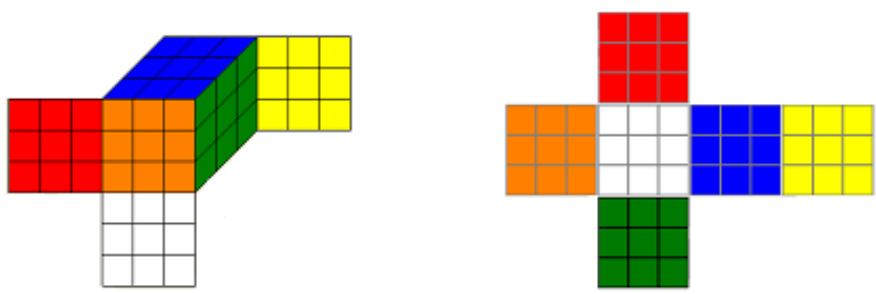

Fig. 3. Unrandom Rubik's Cube

To complete Rubik cube game consisting of 6 sides (layers). Each side consists of 9 small squares. When the Rubik's cube is rotated by the user, the specific "rotation" will move and the color settings of each side will change, see Fig. 4 below:
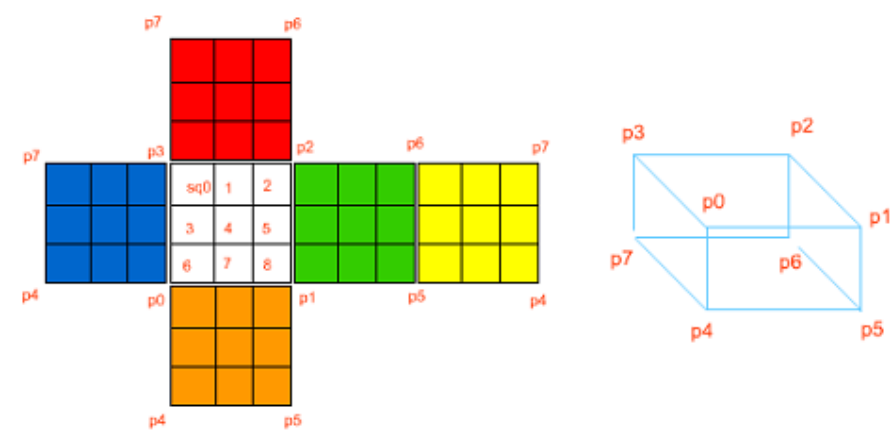

Fig. 4. 6 Side unrandom side Rubik's cube 
Based on Fig. 4, the Rubik's cube completion process is adjusted to the square rotation rules of each color. This square motion is conditioned by coordinates for the Rubik's to be completed. The coordinates are as follows:

1. Data $0=[\mathrm{sq}(4,0), \mathrm{sq}(4,3), \mathrm{sq}(4,6), \mathrm{sq}(1,0), \mathrm{sq}(1,3)$, $\mathrm{sq}(1,6), \mathrm{sq}(5,0), \mathrm{sq}(5,3), \mathrm{sq}(5,6), \mathrm{sq}(3,8), \operatorname{sq}(3,5), \operatorname{sq}(3$, 2)];

2. Data $1=[\mathrm{sq}(4,6), \mathrm{sq}(4,7), \mathrm{sq}(4,8), \mathrm{sq}(2,0), \mathrm{sq}(2,3)$, $\mathrm{sq}(2,6), \mathrm{sq}(5,2), \mathrm{sq}(5,1), \mathrm{sq}(5,0), \mathrm{sq}(0,8), \mathrm{sq}(0,5), \mathrm{sq}(0$, 2)];

3. Data $2=[\mathrm{sq}(4,8), \mathrm{sq}(4,5), \mathrm{sq}(4,2), \mathrm{sq}(3,0), \mathrm{sq}(3,3)$, $\mathrm{sq}(3,6), \mathrm{sq}(5,8), \mathrm{sq}(5,5), \mathrm{sq}(5,2), \mathrm{sq}(1,8), \mathrm{sq}(1,5), \mathrm{sq}(1$, 2)];

4. Data $3=[\mathrm{sq}(4,2), \mathrm{sq}(4,1), \mathrm{sq}(4,0), \mathrm{sq}(0,0), \mathrm{sq}(0,3)$, $\mathrm{sq}(0,6), \mathrm{sq}(5,6), \mathrm{sq}(5,7), \mathrm{sq}(5,8), \mathrm{sq}(2,8), \mathrm{sq}(2,5), \mathrm{sq}(2$, 2)];

5. Data $4=[\mathrm{sq}(3,2), \operatorname{sq}(3,1), \operatorname{sq}(3,0), \operatorname{sq}(2,2), \operatorname{sq}(2,1)$, $\mathrm{sq}(2,0), \mathrm{sq}(1,2), \mathrm{sq}(1,1), \mathrm{sq}(1,0), \mathrm{sq}(0,2), \mathrm{sq}(0,1), \mathrm{sq}(0$, $0)$;

6. Data $5=[\mathrm{sq}(1,6), \mathrm{sq}(1,7), \mathrm{sq}(1,8), \mathrm{sq}(2,6), \mathrm{sq}(2,7)$, $\mathrm{sq}(2,8), \mathrm{sq}(3,6), \mathrm{sq}(3,7), \mathrm{sq}(3,8), \mathrm{sq}(0,6), \mathrm{sq}(0,7), \mathrm{sq}(0$, $8)$;

7. MiddleData $5=[\mathrm{sq}(0,3), \mathrm{sq}(0,4), \mathrm{sq}(0,5), \mathrm{sq}(1,3), \mathrm{sq}(1$, 4), $\mathrm{sq}(1,5), \mathrm{sq}(2,3), \mathrm{sq}(2,4), \mathrm{sq}(2,5), \mathrm{sq}(3,3), \mathrm{sq}(3,4)$, $\mathrm{sq}(3,5)]$;

8. MiddleData $0=[\mathrm{sq}(1,1), \mathrm{sq}(1,4), \mathrm{sq}(1,7), \mathrm{sq}(5,1), \mathrm{sq}(5$, 4), $\mathrm{sq}(5,7), \operatorname{sq}(3,7), \operatorname{sq}(3,4), \operatorname{sq}(3,1), \operatorname{sq}(4,1), \operatorname{sq}(4,4)$, $\mathrm{sq}(4,7)]$

9. MiddleData $3=[\mathrm{sq}(0,1), \mathrm{sq}(0,4), \mathrm{sq}(0,7), \mathrm{sq}(5,3), \mathrm{sq}(5$, 4), $\mathrm{sq}(5,5), \mathrm{sq}(2,7), \mathrm{sq}(2,4), \mathrm{sq}(2,1), \mathrm{sq}(4,5), \mathrm{sq}(4,4)$, $\mathrm{sq}(4,3)]$;

The above step process details can be seen in the Fig. 5 .

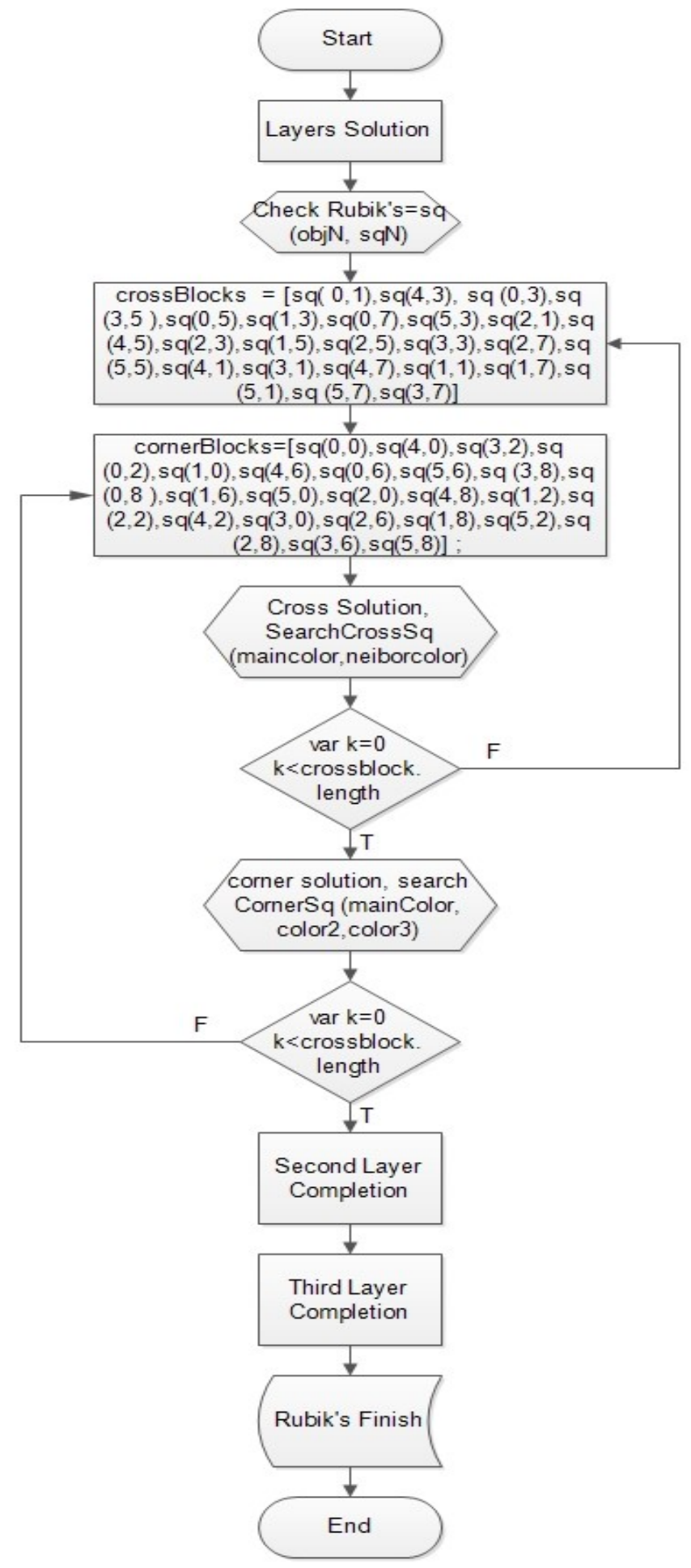

Fig. 5. Flowchart process Rubik's cube solution

\section{RESUlTS AND Discussion}

Application made using Borland Delphi programming language with 3 (three) level of difficulty of randomness to be completed by player, see Fig. 6 . 


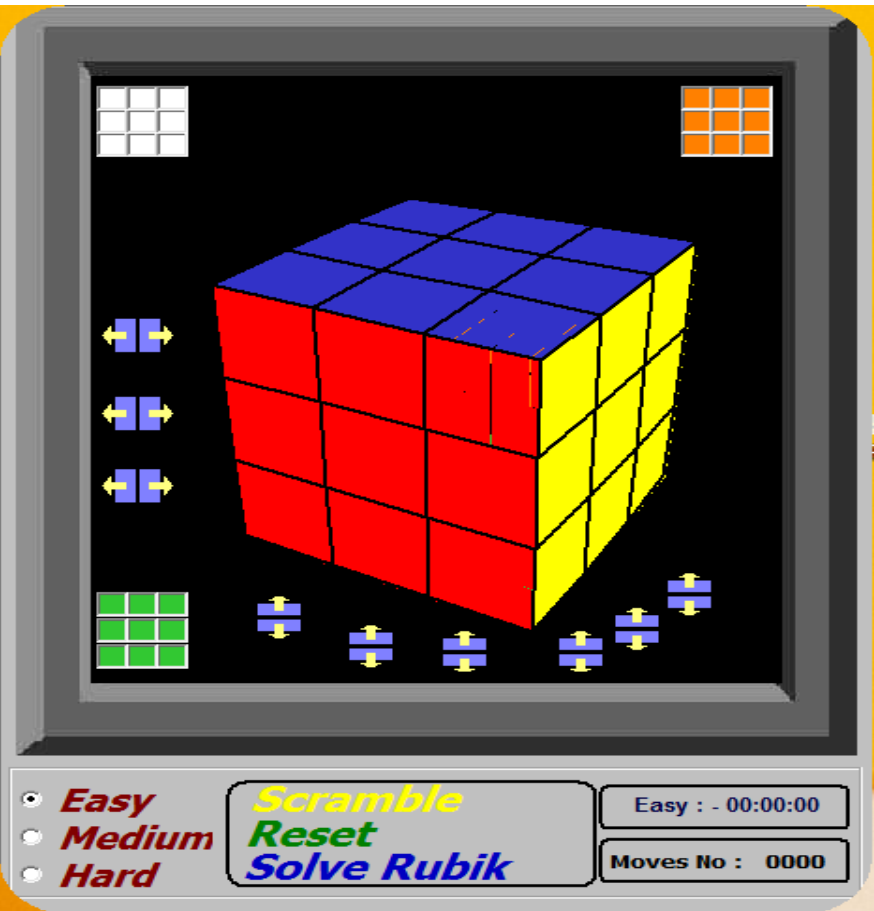

Fig. 6. Rubik's cube application

Fig. 6 is a $3 \times 3$ Rubik's game object created in 3dimensional form where each side can be rotated using available buttons and can also rotate 180 degrees. Experimenting process of Rubik will be random with easy level, see Fig. 7 for result.

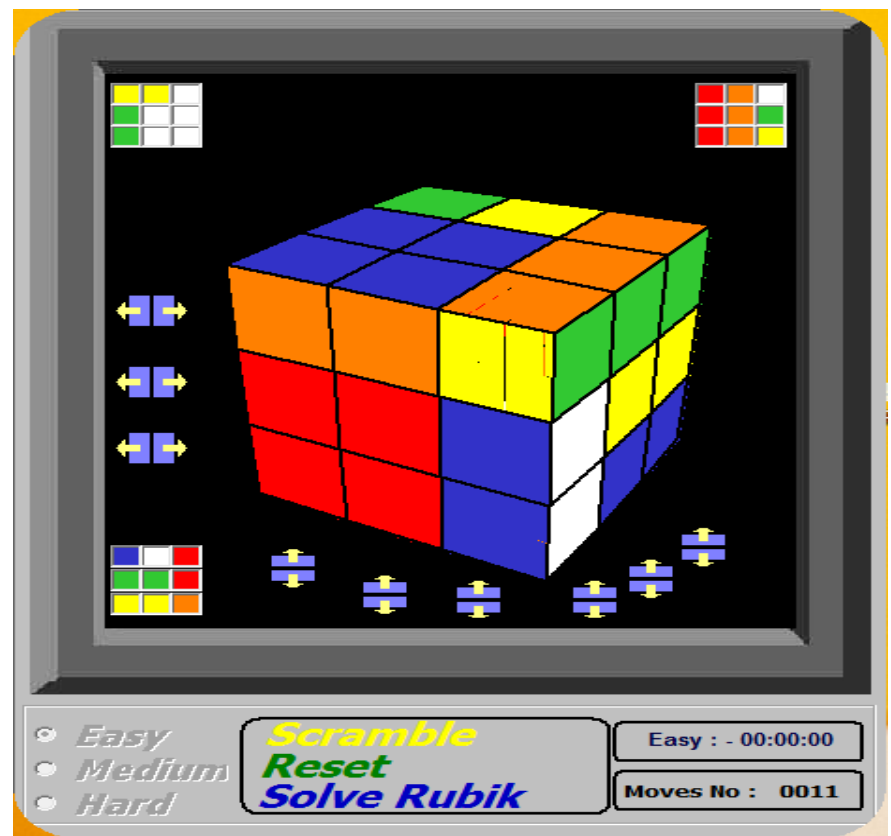

Fig. 7. Rubik's cube with easy scramble

The random Rubik's cube in Fig. 7 also displays step information to complete the Rubik's, in the Fig. 7 it appears that the Rubik's cube can be completed with 11 steps by player or also used Solve Rubik button to complete automatically which can be followed by the beginner player, Fig. 8 .

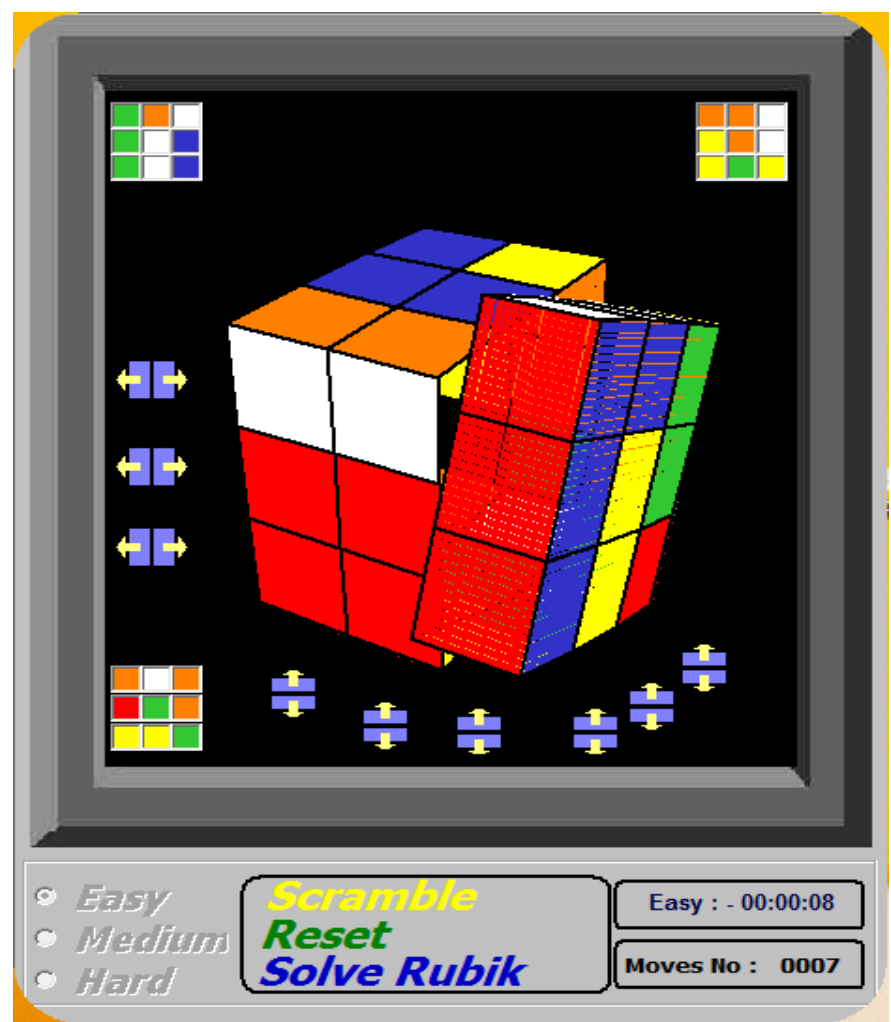

Fig. 8. Solve Rubik's

An experiment performed for each difficulty level are shown in Table I.

TABLE I. EXPERIMENT RESULT

\begin{tabular}{|l|l|l|l|l|}
\hline No & Difficulty & Pattern & $\begin{array}{l}\text { Solution } \\
\text { (Moves) }\end{array}$ \\
\hline 1 & Easy & $\begin{array}{l}11 \text { Moves } \\
\text { with } 4 \\
\text { second to } \\
\text { solve it }\end{array}$ \\
\hline
\end{tabular}


TABLE I. EXPERIMENT RESULT (CONTINUE)

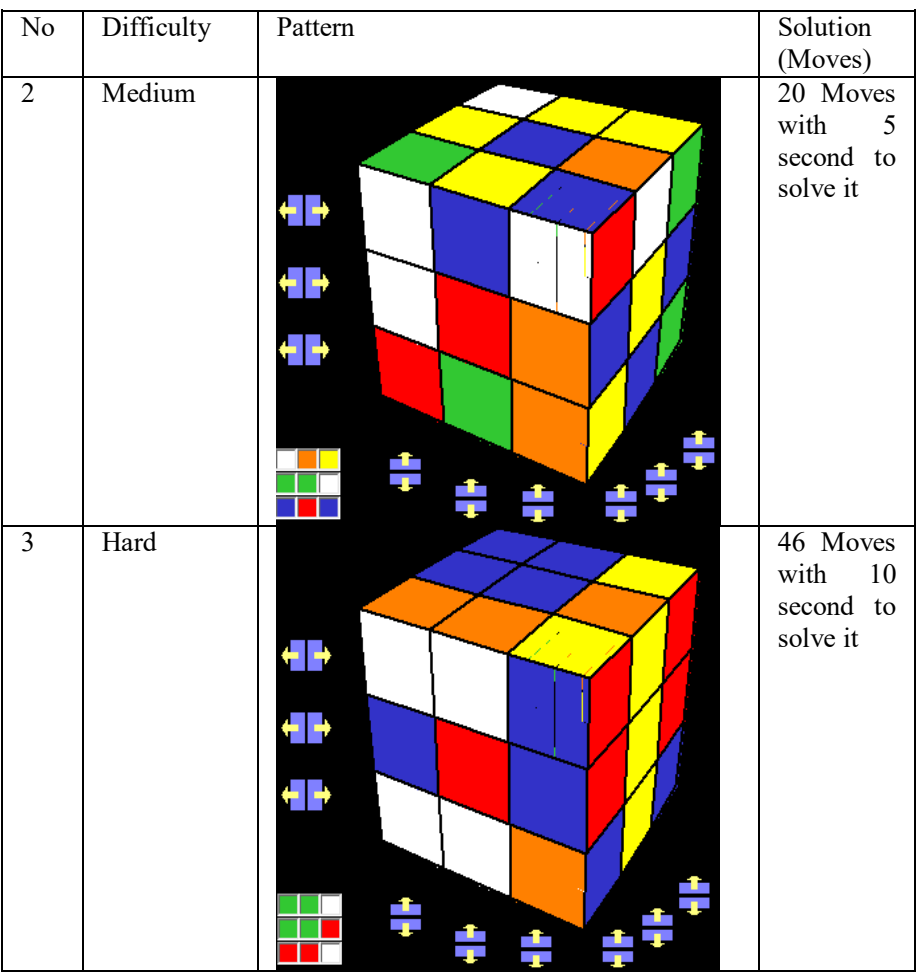

\section{CONCLUSION}

Based on the experiment on Rubik's cube $3 \times 3$ application and with the implementation of a layer by layer (layers) algorithm is known the number of movements that must be done by players and no more Rubik's cube that is difficult to be solved.

\section{REFERENCES}

[1] A. Mueller, "Rubik's Cube For The Computer.," Des. News, vol. 61, no. 4, p. 76, 2006.

[2] E. D. Demaine, M. L. Demaine, S. Eisenstat, A. Lubiw, and A. Winslow, "Algorithms for solving Rubik's cubes," in Lecture Notes in Computer Science (including subseries Lecture Notes in Artificial Intelligence and Lecture Notes in Bioinformatics), 2011, vol. 6942 LNCS, pp. 689-700.

[3] D. Kunkle and G. Cooperman, "Solving Rubik's Cube," Commun. ACM, vol. 51 , no. 4, p. 31, 2008

[4] J. Chen, "Solving Rubik's Cube Using SAT Solvers," arXiv:1105.1436, 2011.

[5] B. Rohrig, "Puzzling Science: Using the Rubik's Cube to Teach Problem Solving," Sci. Teach., vol. 77, no. 9, pp. 54-56, 2010.

[6] S. Loyd and B. Kocka, "A Mathematical Approach To Solving Rubik â $€^{\mathrm{TM}} \mathrm{s}$ Cube," 2005.

[7] D. Kunkle and G. Cooperman, "Twenty-six moves suffice for Rubik's cube," Proc. Int. Symp. Symb. Algebr. Comput., pp. 235-242, 2007.

[8] O. Bergig et al., "Out of the cube: Augmented Rubik's cube," Int. J. Comput. Games Technol., 2011.

[9] H. Samadi and M. R. Daliri, "Solve the Rubik's cube with robot based on non-invasive brain computer interface," in 2014 Iranian Conference on Intelligent Systems, ICIS 2014, 2014.

[10] N. El-Sourani, S. Hauke, and M. Borschbach, "An evolutionary approach for solving the Rubik's cube incorporating exact methods," in Lecture Notes in Computer Science (including subseries Lecture Notes in Artificial Intelligence and Lecture Notes in Bioinformatics), 2010, vol. 6024 LNCS, no. PART 1, pp. 80-89.

[11] B. Barzegar, H. A. Saravi, and S. A. Saravi, "Optimal solutions For Solve Rubik â $€^{\mathrm{TM}}$ s Cube," vol. 5, no. 11, pp. 1225-1235, 2011.

[12] R. E. Korf, "Finding Optimal Solutions to Rubik's Cube Using Pattern Databases," Am. Assoc. Artif. Intell., pp. 700--705, 1997. 